\title{
Implementation of a Shift Register using Pulsed Latch
}

\author{
T.Santosh Kumar, T.Anil Kumar
}

\begin{abstract}
precise This paper proposes a low strength pass sign in planned making use of a beat lock. Supplanting the ace slave turn-flop with a hook decreases manage usage and vicinity. This method settle the breaking factors of the beat lock. making use of hook we are moving statistics to each the developing side and falling edge the usage of a beat clock created utilizing numerous clock turbines. The accompanying pressure go with the flow register is deliberate and executed utilising the Xilinx VIVADO tool.The proposed 256-piece float registers eat an awful lot much less strength and own plenty much less area at the chip than traditional go with the flow registers with turn-flops.
\end{abstract}

Catchphrases - flip-flop, beat clock, beat lock, flow sign on..

\section{INTRODUCTION}

In advanced circuits a drift register is utilized in numerous applications. circulate registers are utilized in numerous programs, as an example, advanced channels, correspondence creditors, image sensor ICs. As of late the facts size keeps on increasing due to the recognition for immoderate facts, the phrase length of the go with the flow check in increments for coping with massive image facts in photograph getting ready ICs. because the word length of the flow join up expands, the strength and challenge of the flow sign up turn out to be huge form contemplations.

The layout of the flow into sign on is fundamental. A nbit go with the flow check in is related to the affiliation $n$ statistics turn lemon. The tempo of the flip-flop is much less good sized than the arena and electricity usage for the cause that there is no circuit amongst flip-flips in the circulate join up. The littlest reminiscence element is reasonably-priced for circulate check in to decrease place and electricity usage. As of overdue, laches have supplanted turn-flounders in numerous VLSI gadgets,due to the fact a hook is lots littler than a few other memory element. Be that as it may, the beat lock can't be implemented inside the skip sign up because of the planning hassle a few of the beat hook.

This paper proposes a how the locks will characteristic and the way we're transferring the facts via by hooks. The flow into check in makes use of few the clock flag by means of preserving apart the hooks to a few amassing shifter registers and making use of greater stockpiling locks.

Revised Manuscript Received on September 10, 2019.

T.Santosh Kumar, Assistant Professor Dept.Of Ece, Cmrit, Hyderabad, Telangana, India.

Dr. T.Anil Kumar, professor and head. dept.of Ece, Cmrit, Hyderabad, Telangana, India.

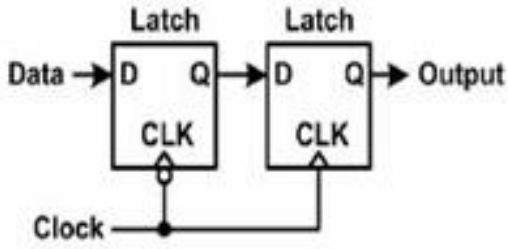

(a)

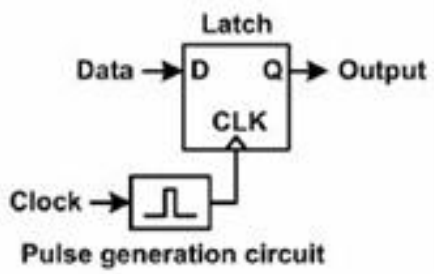

(b)

Fig. 1 (an) ace slave flip-flop (b) beat Latch

\section{A. Proposed circulate check in:}

An ace slave turn-flop is synthetic the usage of hook in fig. 1 (a) may be supplanted with a beat hook that has a lock and a beat test sign as in Fig. 1 (b). All hook uses heartbeat clock generator circuit for beat clock signal. therefore, the proposed move register vicinity and beat lock energy decreases sort of $50 \%$ of the turn-flops. Beat hook alternate is an attractive answer for low field and coffee power programs.

The beat lock cannot be utilized legitimately in circulate enlists because of the time types of clock signal, The circulate join up incorporates of sure hooks and a beat clock signal (CLK_pulse). The yield signal of the primary lock refreshes successfully in view that the information sources signal of the primary hook (IN) is everyday within the path of the clock beat width. Be that as it is able to, the following hook has a doubtful yield sign (Q2) due to the fact its facts sign (Q1) modifications at some point of the clock beat width.

One solution for the making plans kinds is to encompass deferred circuits among locks; The yield signal of the hook is postponed and arrives at the subsequent lock after the clock beat. The yield signal of the primary and second locks updates in the direction of the clock beat width, but the statistics signal of the second one and 0.33 hooks emerge as same to the yield sign of the primary and 2nd locks after the clock beat. ultimately, all of the hook have consistent

Published By:

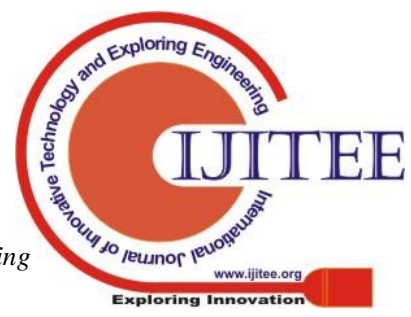


information indicators at a few stage in the clock beat and there can be no planning problem among the lock. Be that as it may, the put off circuit reasons giant location and power overhead.

any other association is to utilize numerous postponed beat clock indicators. The deferred beat clock sign are produced whilst a clock sign reviews delay circuits the numerous clock heartbeats will created. each lock makes use of a beat clock signal that is deferred with the aid of way of the beat check sign applied in its next hook. in the end, every lock adjustments the information when its subsequent hook refreshes the statistics. subsequent to associating the put off circuit each hook has a comparable contribution throughout the clock beat and there may be no time variety between the lock. Be that as it is able to, this association likewise calls for numerous get rid of circuits.

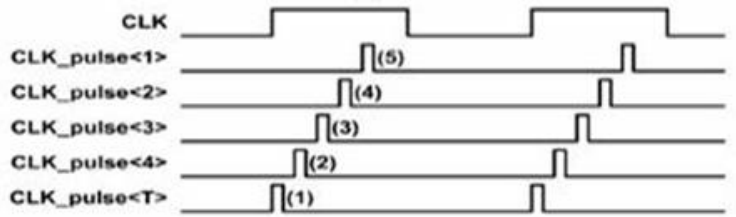

determine 2: diverse postponed beat clock indicators

five numerous non-covers deferred clock sign are created thru the postponed beat test generator in Fig. four. The succession of the beat take a look at sign is in the opposite request of the locks. before everything, the clock signal CLK_pulse $<\mathrm{t}>$ adjustments the lock records $\mathrm{t} 1$ from this autumn. And after that, the clock signs CLK_pulse<1:4> changes the four hook information from this autumn to q1 successively. The hooks $\mathrm{q} 2-\mathrm{q} 4$ get facts from their locks Q1-Q3 however the number one hook Q1 receives facts from the contribution of the flow into take a look at in (commotion). The duties of the opportunity gathering movement registers are equal to that of the flow into sign in \#1 apart from that the number one lock gets data from the capacity hook within the past gathering movement sign in.

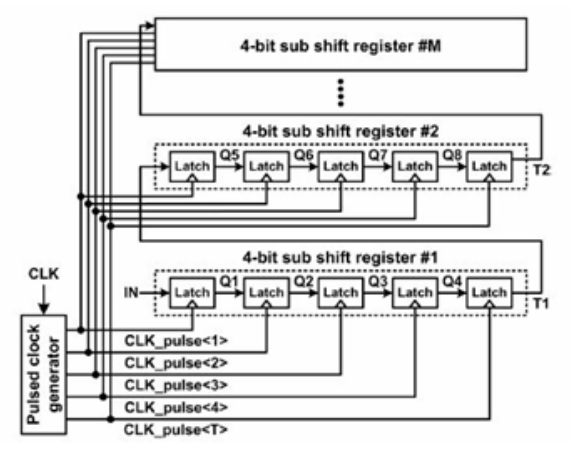

Figure 3: proposed shift register

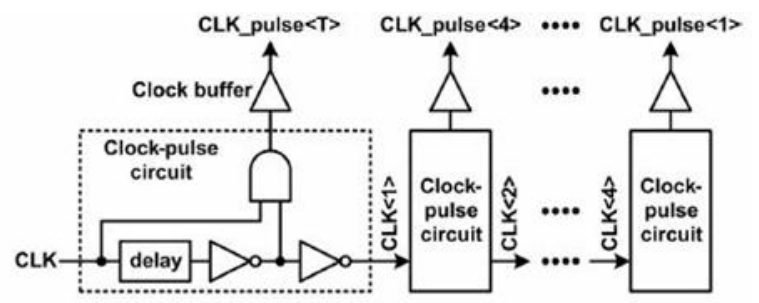

\section{Discern four: more than one now not on time pulsed clock signal generator}

\section{A. Vicinity optimization:}

The proposed shift take a look at in reduces the huge form of pulsed clock signs drastically, however it'll boom the good sized form of latches because of the more brief storage latches. As shown in Fig.4 each pulsed clock sign is generated in a pulsed clock-circuit consisting a postpone circuits and an and gate. At the same time as an n-bit shift join up is cut up into suitable sufficient-bit sub shift registers, the tremendous type of no longer on time pulsed clock circuits is right enough +1 and the range of latches is $n+n / k$. An top notch enough-bit sub shift sign in which encompass okay+1 latches calls for ok +1 pulsed clock signs. The massive fashion of $\mathrm{m}$ sub shift registers becomes $\mathrm{n}$ /proper sufficient, each sub shift sign up has a quick garage latch. Consequently, n/ok latches are added for the short garage latches.

The numbers of latches and pulsed clock signal circuits trade normal with the records duration of the grouped shift take a look at in (adequate). Good enough is chosen with the resource of thinking about the vicinity, electricity consumption, tempo.

The optimization of region can be finished as follows. At the same time as the region of the circuit are normalized with a latch and the location of a latch and a pulsed clock circuit generator are 1 and $\alpha \mathrm{A}$, respectively. The general vicinity will become $\quad \mathrm{A}=\alpha \mathrm{A}$ (properly

enough+1)+N(1+l/okay).

The maximum beneficial $\mathrm{ok}(=(\mathrm{n} / \mathrm{ok}) \mathrm{half}$ of $)$ for the minimal vicinity is acquired from the primary-order differential equation of the whole region(zero $=\alpha \mathrm{A}-\mathrm{N} / \mathrm{okay})$. An integer adequate for the minimal location is chosen as a divisor of $n$, that is nearest to $(\mathrm{N} / \alpha \mathrm{A}) 1 / 2$ of.

\section{Strength optimization:}

Electricity optimization is similar to assignment optimization. Energy is in particular fed on in latches and clock-pulse circuits. Each latch consumes energy for information transition and clock loading. Whilst the circuit power is normalized with a latch, the electricity consumed with the beneficial aid of a latch and a clock-pulse circuit is 1 and $\alpha \mathrm{P}$, respectively. Not unusual power consumption is likewise $\mathrm{P}=\alpha \mathrm{P}($ good enough +1$)+\mathrm{N}(1+1 /$ correct enough $)$.

For minimum power an integer adequate is selected as a denominator, it actually is closest $(\mathrm{N} / \alpha \mathrm{P}) 1 / 2$ The popular period of the clock buffers is decided thru using the overall clock loading of latches. Despite the fact that the sort of latches will growth from $\mathrm{N}$ to $\mathrm{N}(1+1 /$ adequate $)$, the increment ratio of the clock buffers is small. The amount of clock buffers is . As adequate will growth, the dimensions of a clock buffer decreases in percent to due to the truth the sizeable form of latches connected to a clock buffer is ( $\mathrm{M}=\mathrm{N} / \mathrm{ok}$ ) proportional to $1 / \mathrm{ok}$. Therefore, the whole period of the clock buffers will boom slightly with developing and the effect of the clock buffers may be left out for deciding on.

Outcomes: 
International Journal of Innovative Technology and Exploring Engineering (IJITEE)

ISSN: 2278-3075, Volume-8, Issue-11S2, September 2019

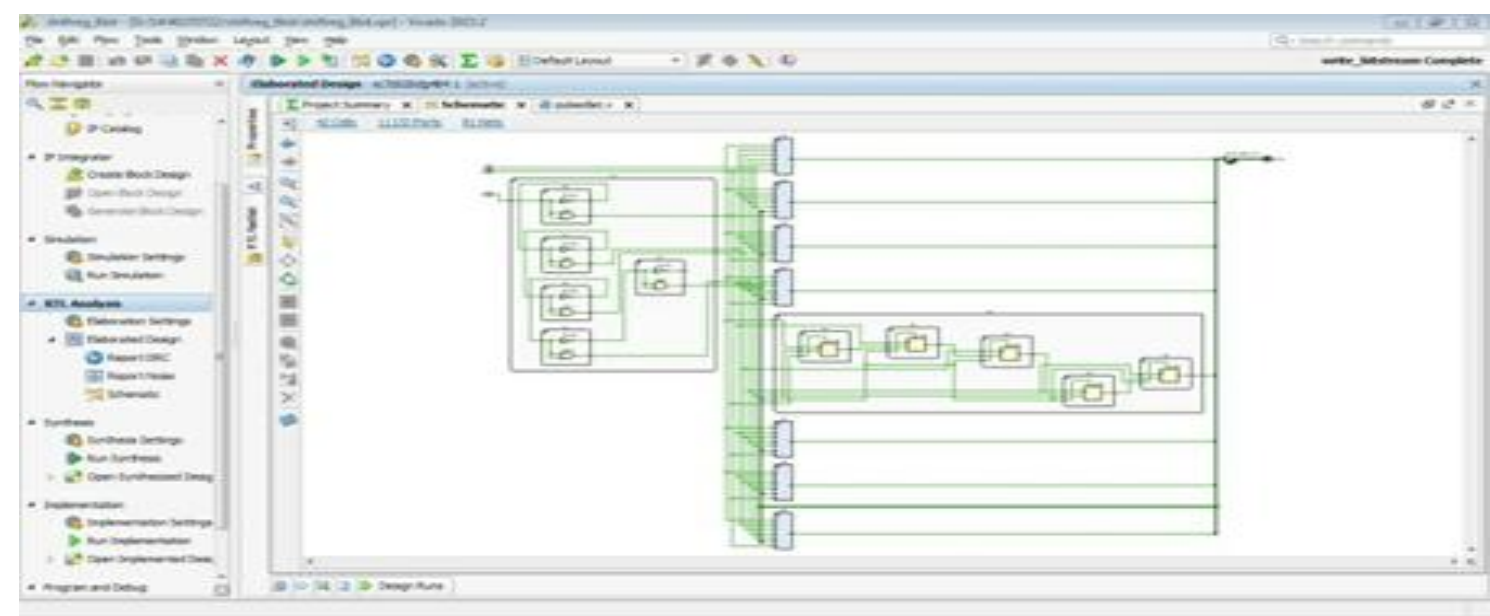

Figure 5: Proposed shift register schematic

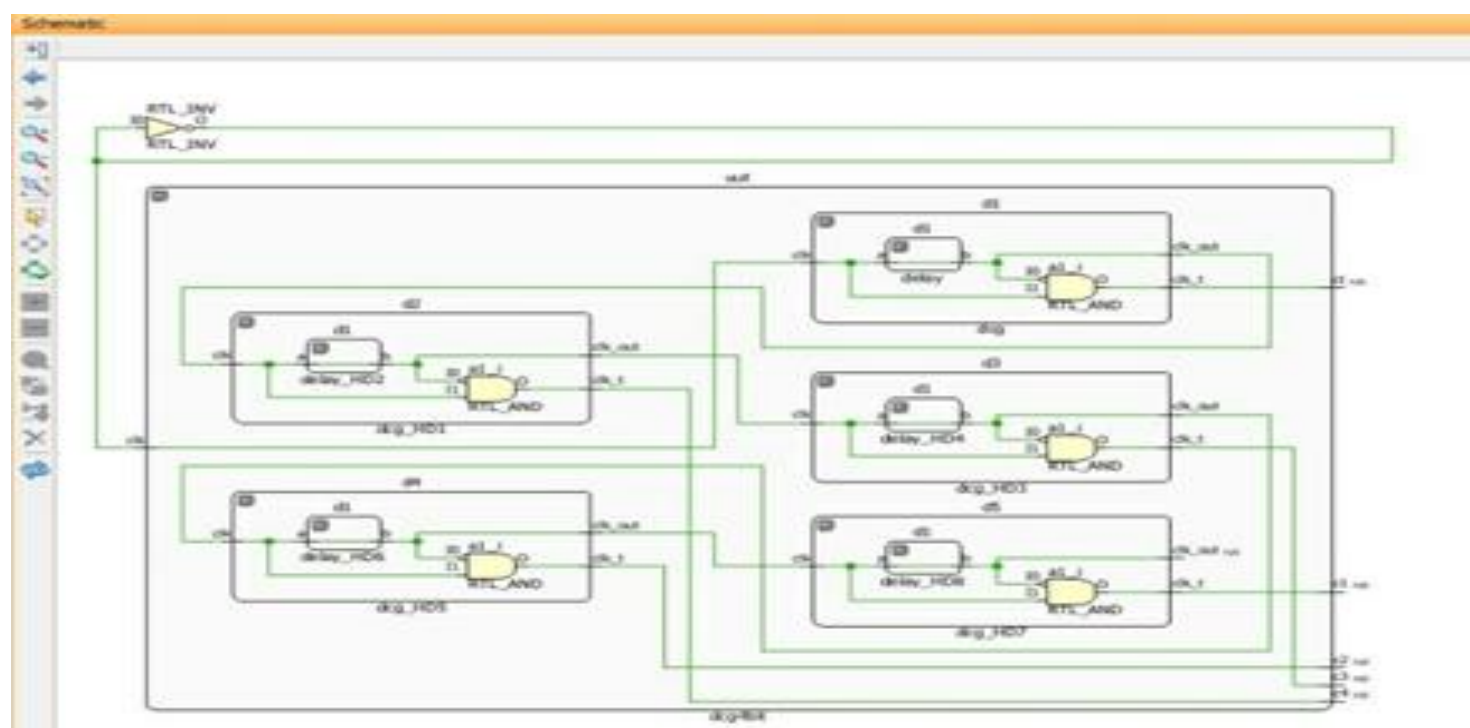

Figure 6: Schematic of multiple delayed clock generator

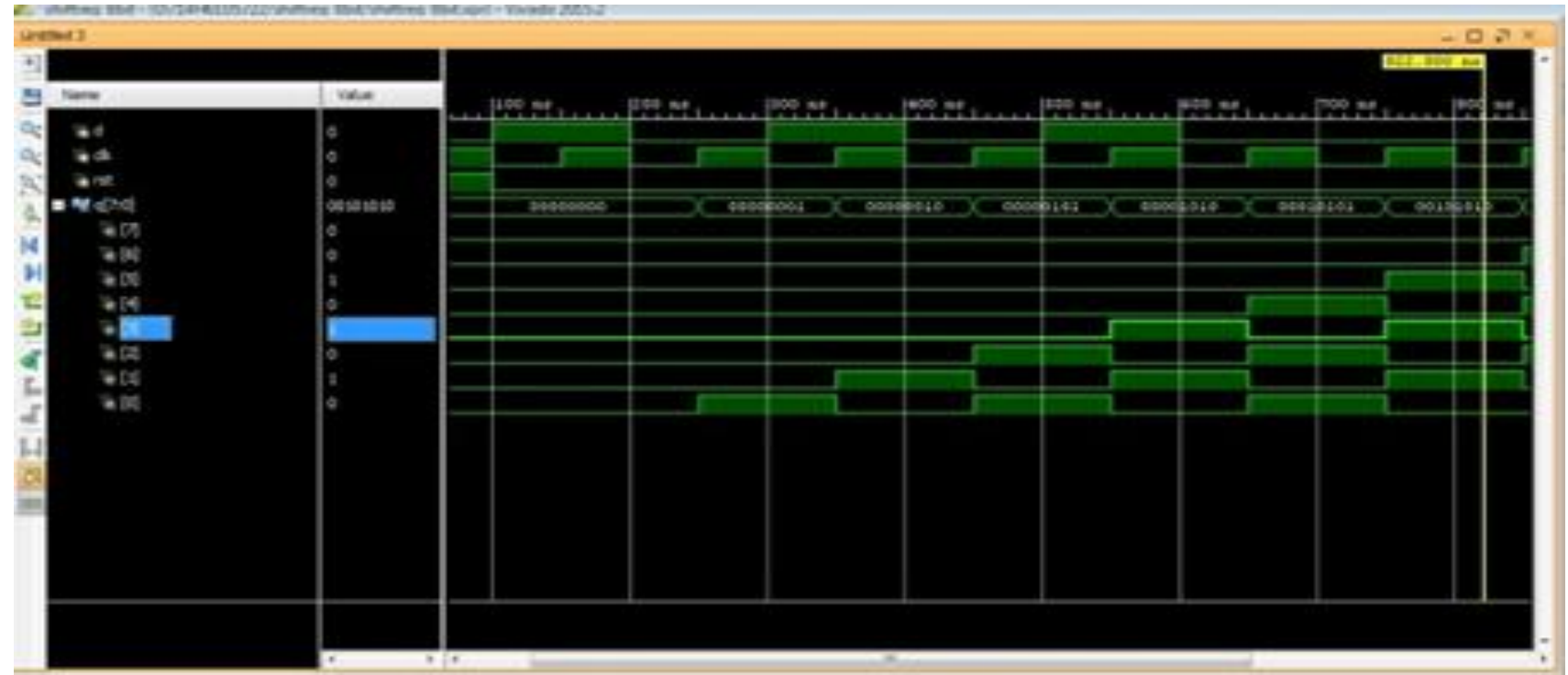

Figure 7: proposed Shift register output waveform

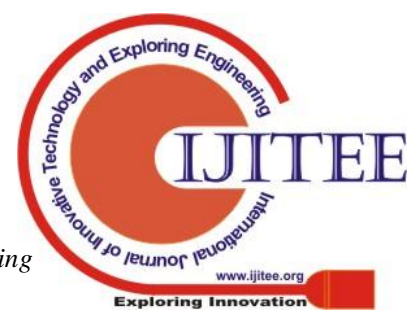




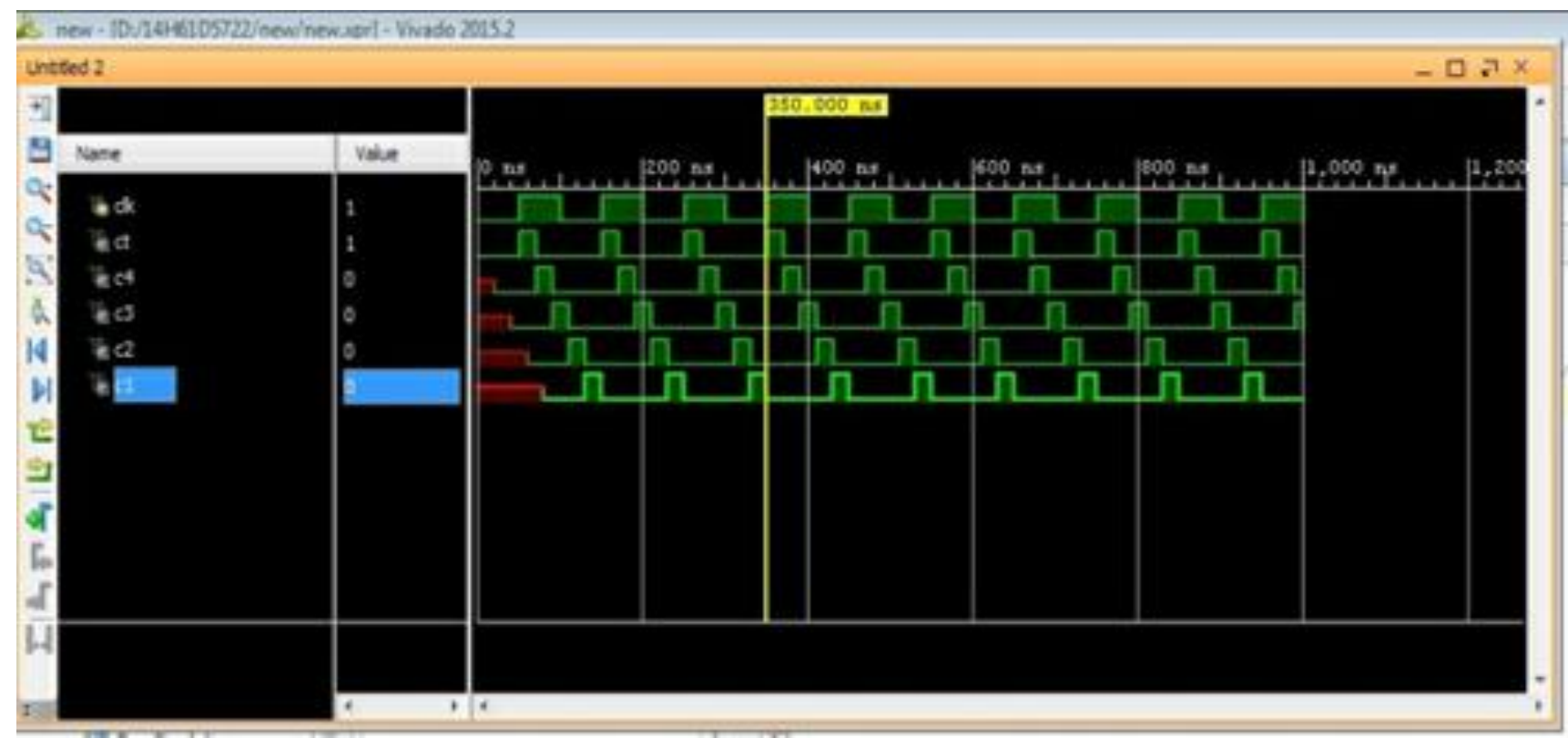

Determine 8: Waveform of more than one no longer on time pulsed clock signal

CASE take a look at inside the prevailing examine,the proposed shift join up is designed via manner of using the Verilog HDL in VIVADO tool and carried out on Zynq Board. The front prevent designing of the shift check in is pretty clean. The replace of the turn-flops with Latches as a stop end result half of of of of of the area and power consumption is reduced. The fabrication of the proposed Shift join up IC takes low price.

\section{SURRENDER \& RESULTS}

This paper proposed a low-strength shift sign in the utilization of pulsed latch. Shift check in reduces place and electricity intake with the useful aid of converting turn-flops with laps. The time trouble amongst latch is solved with the resource of manner of way of using numerous not on time pulsed clock symptoms in preference to a unmarried pulsed clock sign.A small style of pulsed clock signs are used by grouping latchis to severa sub-shifter registers and the usage of greater short garage latch. Implemented the use of a 256bit shift take a look at in Vivado Zynq Board in form technique. The proposed shift be a part of up saves $37 \%$ region and $404 \%$ strength compared to the traditional shift sign up with turn-flops.

\section{REFERENCES}

1. S.-H. W. Chiang and S. Kleinfelder, "Scaling and format of a sixteen-mega pixel CMOS picture sensor for electron microscopy," in Proc. IEEE Nucl. Sci. Symp. Conf. File (NSS/MIC), 2009, pp. 1249-1256.

2. V..Stojanovic'sand V..Oklobdzija's , "Comparative evaluation of maintain near-slave latches and turn-flops for immoderate-ordinary performance and coffeeelectricity structures" IEEE J. Strong-us of a Circuits, vol. 34, no. 4, pp. 536-548, Apr.1999.

3. V. Stojanovic and V. Oklobdzija, "Comparative evaluation of masterslave latches and turn-flops for excessive-popular accepted standard overall performance and espresso-strength systems," IEEE J. Sturdy-kingdom Circuits, vol. 34, no. Four, pp. 536-548, Apr.1999
4. H.. Yamasaki's \& T. Shibata's , "A real-time photofunction-extraction and vector-generation vlsi using arrayed-shift-sign in shape," IEEE's J. Robust-nation's Circuit's, Vol . V2, No. Nine,pP- 2046/2053, September 2007.

5. S. Li, H. S. Matis, N. H. Xuong, S. Kleinfelder, "Modeling and assessment of Charged-Particle CMOS photograph sensor arrays," IEEE Transactions on Nuclear technology, Vol. Fifty six, No. Three, detail 2, June 2009, pp. 1062-1068.

6. S. H. Unger et al., "Clocking schemes for immoderatepace virtual structures," IEEE Trans. Comput., vol. C-35, pp. 880-895, Oct. 1986.

7. V. Stojanovicet al., "Comparative evaluation of graspslave latches and turn-flops for high-widespread usual performance and espresso-power structures," IEEE J. Strong-u.S.A. Circuits, vol. 34, pp. 536-548, Apr. 1999.

8. V. Stojanovicet al., "A unified approach in the evaluation of latches and flip-flops for low-power systems," in Int. Symp. Low-energy Electronicsand design, Monterey, CA, Aug. 1998, pp. 227-232.

9. T. Sakurai et al., "Low-strength circuit format for multimedia CMOS VLSIs," in Proc. Synthesis Sys. Integration combined Technol. (SASIMI), Nov. 1996, pp. Three-10.

10. H. Partoviet al., "go with the flow-thru latch and aspectprompted flip-flop hybrid elements," in Int. Robust-usa Circuits Conf.E, Dig. Of Tech. Papers, Feb.1996, pp. 138-139.

11. F. Klass, "Semi-dynamic and dynamic flip-flops with embedded right judgment,"inSymp. On VLSI Circuits, Dig. Of Tech. Papers, June 1998, pp. 108-109.

12. Matsui et al., "one hundred $\mathrm{MHz} 13 \mathrm{~mm}$ 2-D DCT macrocell the usage of revel in amplifying pipeline turnflop scheme," IEEE J. Strong-country Circuits, vol. 29, pp. 1482-1490, Dec. 1994. 